(C) World Scientific, Mod. Phys. Lett. A 17 no. 21 (2002) 1377-1381

Los Alamos arXiv gr-qc/0205085 v2

\title{
ON ARITHMETIC DETECTION OF GREY PULSES WITH APPLICATION TO HAWKING RADIATION
}

\author{
HARET C. ROSU \\ hcr@ipicyt.edu.mx \\ Dept. of Applied Mathematics, IPICyT, Apdo Postal 3-74 Tangamanga, San Luis Potosí, MEXICO \\ MICHEL PLANAT \\ planat@lpmo.edu \\ Laboratoire de Physique et Métrologie des Oscillateurs du CNRS, 25044 Besançon Cedex, FRANCE
}

\begin{abstract}
Micron-sized black holes do not necessarily have a constant horizon temperature distribution. The black hole remote-sensing problem means to find out the 'surface' temperature distribution of a small black hole from the spectral measurement of its (Hawking) grey pulse. This problem has been previously considered by Rosu, who used Chen's modified Möbius inverse transform. Here, we hint on a Ramanujan generalization of Chen's modified Möbius inverse transform that may be considered as a special wavelet processing of the remote-sensed grey signal coming from a black hole or any other distant grey source.
\end{abstract}

Keywords: Möbius transform, Ramanujan sums, grey-body, Hawking radiation, black hole

\section{INTRODUCTION}

There may exist black holes in the micron size range carrying on some externaldistribution of matter. Theoretical examples are (i) down-scaled Weyl black holes, ${ }^{1}$ for which the metric potentials are solutions of polar Laplace equations (ii) black holes with Einstein shells,2 (iii) primordial black holes (PBH) and/or any mini black hole hovering through the universe and carrying on some matter distributions, (iv) hairy black holes, 3 with additional conserved quantum numbers beyond those allowed by the classical no hair theorems and dirty black holes in the sense of Visser, holes in interaction with various classical fields, for which the Hawking temperature appears to be supressed relative to the vacuum black holes of equal area. In some cases, for small enough black holes, the external distribution of matter can be of such a kind as to disturb only slightly the pure horizon Hawking radiation and consequently from the praxis standpoint we have a grey-body radiation problem.

Hawking radiation by itself is distorted with respect to a pure black-body spectrum, especially in the low frequency regime due to a grey-body factor usually identified with the square of the absorption amplitude for the mode. $\mathrm{A}$ A useful work on the nature of the grey body problem for black holes has been written by Schiffer 1

In this letter, we first review the grey body inverse problem and the modified Möbius inverse transform (Chen's transform) in sections 2 and 3, respectively 
In section 4, we hint on a possible Ramanujan extension of Chen's transform with possible application to small black holes and the cosmological background radiation.

\section{INVERSE GREY-BODY PROBLEM}

Planck's law provides the analytical formula for the emitted power spectrum from black body sources. In laboratory physics the emitted power spectrum is also called spectral brightness, or spectral radiance of the black body radiation. The latter notion is used in radiometry to characterize the spectral properties of the source as a function of position and direction from the source. For point, i.e., far away, grey sources the total radiated power spectrum, also called radiant spectral intensity is

$$
W(\nu) \sim \int_{0}^{\infty} A(T) B(\nu, T) d T,
$$

where

$A(T)$ is the area temperature distribution of the grey body,

$B(\nu, T)$ is the Boltzmann-Planck occupation factor.

Finding out $A(T)$ at given $W(\nu)$ is known as the inverse grey-body problem. may be known either experimentally or within some theoretical model. This inverse problem was solved in principle by Bojarski, coldness parameter $u=h / k T$, and an area coldness distribution $a(u)$, as more convenient variables than $T$ and $A(T)$ to get an inverse Laplace transform of the total radiated power. The coldness distribution is obtained as an expansion in this Laplace transform. Explicitly, the total grey power spectrum is rewritten as an integral over the coldness variable

$$
W(\nu)=\frac{2 h \nu^{3}}{c^{2}} \int_{0}^{\infty} \frac{a(u)}{\exp (u \nu)-1} d u
$$

and furthermore as

$$
W(\nu)=\frac{2 h \nu^{3}}{c^{2}} \int_{0}^{\infty} \exp (-u \nu)\left[\sum_{n=1}^{\infty}(1 / n) a(u / n)\right] d u .
$$

Therefore the sum under the integral that we shall denote by $f(u)$ is the Laplace transform of $g(\nu)=\frac{c^{2}}{2 h \nu^{3}} W(\nu)$ and the inverse Laplace transform of $g$ will provide the sought coldness distribution.

Despite the formal mathematical solution the inverse grey body problem is unstable for most numerical implementations, i.e., it belongs to the broad class of ill-posed inverse problems. 10 


\section{MODIFIED MÖBIUS TRANSFORM (MMT)}

Chen 11 obtained $a(u)$ by means of the so-called modified Möbius transform ( MMT) of $f(u)$

$$
a(u)=\sum_{n=1}^{\infty} \frac{\mu(n)}{n} f(u / n) .
$$

To understand Eq. (4) we recall a few basic results from the theory of numbers 12 The Möbius expansion refers to special sums over prime factor divisors, (d-sums) running over all the prime factors of $n, 1$ and $n$ included, of any function $f(n)$ defined on the positive integers

$$
S_{f}(n)=\sum_{d \mid n}^{n} f(d) .
$$

The remarkable fact in this case is that the last term of the sum can be written in turn as a sum over the $S_{f}$ arithmetical functions. The latter sum is called the inverse Möbius transform (or the Möbius d-sum) of $f$

$$
f(n)=\sum_{d \mid n}^{n} \mu(d) S_{f}(n / d),
$$

in which the d-sum $S_{f}(n)$ becomes the first term of the Möbius d-sum, and where $\mu(d)$ is the famous Möbius function. Since at the left hand side of (6) one has only a term of a d-sum whereas on the right hand side there is a sum of d-sums there is clear overcounting, unless the Möbius function is sometimes either naught or negative. The partition of the prime factors of $n$ implied by the Möbius function is such that, by definition, $\mu(1)$ is $1, \mu(n)$ is $(-1)^{r}$ if $n$ includes $r$ distinct prime factors, and $\mu(n)$ is naught in all the other cases. In particular, all the squares have no contribution to the inverse Möbius transforms. That is why the integers selected by the Möbius function are also called square-free integers.

Chen's MMT means to apply such an inversion of finite sums to infinite summations, and to ordinary functions of real continuous variable(s). MMT means that if

$$
y_{1}(x)=\sum_{n=1}^{\infty} y_{2}(x / n),
$$

then

$$
y_{2}(x)=\sum_{n=1}^{\infty} \mu(n) y_{1}(x / n) .
$$

For the inverse grey-body problem, $y_{1}(u)=u f(u)$ and $y_{2}(u)=u a(u)$. So, one can get the coldness distribution by multiplying the Laplace transform of the total power spectrum by the coldness parameter, and then applying the MMT. 


\section{RAMANUJAN GENERALIZATION OF CHEN'S TRANSFORM}

Ramanujan sums are well known in number theory but only recently some physical applications have been suggested.13 They are of the form

$$
c_{q}(m)=\sum_{p=1}^{q} \cos (2 \pi m p / q),
$$

with irreducible fractions $p / q$. The sums are quasiperiodic in $m$ and aperiodic in the denominator $q$. They are a generalization of the Möbius function since $c_{q}(m)=\mu(q)$ whenever $q$ and $m$ are coprimes. The MMT for black holes in the Ramanujan notation will be

$$
[u a(u)]_{(1)}=\sum_{q=1}^{\infty} c_{q}(1) u f(u / q),
$$

but the point is that many other Ramanujan inverse transforms can be introduced through

$$
[u a(u)]_{(i)}=\sum_{q=1}^{\infty} c_{q}(i) u f(u / q),
$$

for integers $i>1$ and $(q, i)=1$. One can see that a sequence of two-dimensional analyses $(i, q)$ of the signal are available in this approach similarly to the timefrequency analysis that is so characteristic to wavelets.

As an example, for micron-sized Schwarzschild black holes $\left(M \sim 10^{24} \mathrm{~g}\right)$, no known massive particles are thermally emitted, and according to the calculations of Page, 14 about $16 \%$ of the Hawking flux goes into photons, the rest being neutrino emission. Let us consider these black holes as grey objects of the following two classes (i) by their own, 3 and (ii) of the Weyl type. The coldness parameter will be in the first case

$u_{S}=\frac{1}{\nu} \ln \left(1+\frac{e^{\beta_{h} \hbar \omega}-1}{\Gamma(\omega)}\right)$, where $\beta_{h}$ is the horizon inverse temperature parameter, and $\Gamma(\omega)$ is the penetration factor of the curvature and angular momentum barrier around the black hole, 3 whereas in the latter case $u_{S}=h / k T_{d}$, where $T_{d}$ can be considered as an effective horizon temperature of the distorted black holes $T_{d}=$ $(8 \pi M)^{-1} \exp (2 \mathcal{U})$, where $\mathcal{U}$ is given in the work of Geroch and Hartle. With the coldness parameter at hand one can apply the aforementioned number methods for the black hole emissivity problem.

Before closing we mention that an alternative viewpoint on MMT developed by Hughes et al, ${ }^{5}$ in terms of Mellin transform and Riemann's $\zeta$ function is also extremely interesting if one takes into account that the Bekenstein-Mukhanov spectrum, 16 could be consideredas the eigenvalue problem of relativistic Schroedinger equations in finite differences. 17

Thus, it appears that a richer information content of the spectrum of important astrophysical signals that may reveal hidden discrete features can be obtained by extensive use of number theoretical techniques. The cosmological background radiation signal can be studied by the same approach and with the same aim. There 
are claims by Hogan 18 that inflationary perturbations display discreteness not predicted by the standard field theory and that this discreteness may be observable in cosmic background anisotropy.

1. H. Weyl, Ann. Phys. 54 (1917) 117; for recent works, see R. Geroch and J. Hartle, J. Math. Phys. 23 (1982) 680; J.P.S. Lemos and P.S. Letelier, Phys. Rev. D49 (1994) 5135, and references therein.

2. A. Einstein, Ann. Math. 40 (1939) 922. For recent papers see P.R. Brady, J. Louko, and E. Poisson, Phys. Rev. D44 (1992) 1891; G.L. Comer, D. Langlois, and P. Peter, Class. Quantum Grav. 10 (1993) L127; G.L. Comer and J. Katz, Class. Quantum Grav. 10 (1993) 1751.

3. M.J. Bowick et al, Phys. Rev. Lett. 61 (1988) 2823.

4. M. Visser, Phys. Rev. D46 (1992) 2445.

5. J.D. Bekenstein, Phys. Rev. Lett. 70 (1993) 3680; C.F.E. Holzhey and F. Wilczek, Nucl. Phys. B380 (1992) 447; V. Balasubramanian and F. Larsen, Nucl. Phys. B495 (1997) 206; J.M. Maldacena and A. Strominger, Phys. Rev. D55 (1997) 861.

6. M. Schiffer, Gen. Rel. Grav. 27 (1995) 1, and references therein.

7. H.C. Rosu, Mod. Phys. Lett. A13 (1998) 695.

8. For a concise review, see A. Lakhtakia, Mod. Phys. Lett. B5 (1991) 491.

9. N.N. Bojarski, IEEE Trans. Antennas Propag. 30 (1982) 778.

10. Xin Tan et al, J. Opt. Soc. Am. A11 (1994) 1068.

11. N.X. Chen, Phys. Rev. Lett. 64 (1990) 1193, 3202(E); J. Maddox, Nature 344 (1990) 377 .

12. A. Baker, A Concise Introduction to the Theory of Numbers, (Cambridge University Press, Cambridge 1984).

13. M. Planat, Ramanujan sums for signal processing of low frequency noise, contribution at IEEE International Frequency Control Symposium, New Orleans, USA, (29-31 May 2002); M. Planat, H.C. Rosu, S. Perrine, Arithmetical chaology and the signatures of $1 / f$ noise, contribution at TH-2002, Paris, (22-27 July 2002).

14. D.N. Page, Phys. Rev. D13 (1976) 198.

15. B.D. Hughes, N.E. Frankel, B.W. Ninham, Phys. Rev. A42 (1990) 3643.

16. J.D. Bekenstein, V.F. Mukhanov, Phys. Lett. B360 (1995) 7.

17. V.A. Berezin, A.M. Boyarsky, A. Yu. Neronov, On the spectrum of relativistic Schroedinger equation in finite differences, gr-qc/9902028.

18. C.J. Hogan, Holographic discreteness of inflationary perturbations, Phys. Rev. D (2002) to appear, astro-ph/0201020. 